\title{
METHICILLIN AND VANCOMYCIN RESISTANCE AMONG STAPHYLOCOCCUS AUREUS STRAINS ISOLATED FROM PATIENTS ATTENDING TERTIARY CARE HOSPITAL IN EASTERN BIHAR
}

\author{
Randhir Kumar ${ }^{1}$, Krishan Nandan'2, Sangeeta Dey33, Dhananjay Kumar ${ }^{4}$, Dharmendra Singh ${ }^{5}$ \\ ${ }^{1}$ Senior Resident, Department of Microbiology, IGIMS, Patna. \\ ${ }^{2}$ Associate Professor, Department of Microbiology, Katihar Medical College, Katihar. \\ ${ }^{3}$ Professor and HOD, Department of Microbiology, Katihar Medical College, Katihar. \\ ${ }^{4}$ Assistant Professor, Department of Microbiology, Katihar Medical College, Katihar. \\ 5Tutor, Department of Microbiology, Katihar Medical College, Katihar.
}

\section{ABSTRACT}

\section{BACKGROUND}

Staphylococcus aureus is notorious for its ability to become resistant to antibiotics. MRSA emerged as nosocomial pathogen in the early 1960s. Methicillin Resistant Staphylococcus aureus (MRSA) are implicated in serious infections and nosocomial infection outbreaks. These strains show resistance to a wide range of antibiotics, thus limiting the treating options to very few agents such as vancomycin and teicoplanin. Vancomycin has been regarded as the first-line drug for treatment of MRSA. At the same time, there has been an increase in the use of this antibiotic for other infections as well. This has further lead to an increase in the number of both Vancomycin Intermediate Staphylococcus aureus (VISA) and Vancomycin Resistant Staphylococcus aureus (VRSA).

Aims- To determine the presence of MRSA and VRSA among staphylococcal isolates in Eastern Bihar.

\section{MATERIALS AND METHODS}

A total of 10806 samples from patients attending inpatient and outpatient departments from January 2011 to April 2013 were included in the study. Samples were processed as per standard protocol and antibiotic susceptibility testing was done by modified Kirby-Bauer method. Isolates were tested by disc diffusion using oxacillin disc $1 \mu \mathrm{g}$, cefoxitin disc $30 \mu \mathrm{g}$ and by agar dilution for MRSA. VRSA isolates were tested using $30 \mu \mathrm{g}$ vancomycin disc. MIC of vancomycin to Staphylococcus aureus was determined by agar dilution method.

\section{RESULTS}

Out of a total of 633 Staphylococcus aureus isolates, 22.4\% were found to be methicillin resistant, 9.95\% were VISA and 3.79\% were VRSA. Results of oxacillin agar dilution method were in concordance with the cefoxitin disc diffusion method in detecting MRSA strains. All VISA strains were sensitive to linezolid and all VRSA were sensitive to imipenem. $88.7 \%$ and $87.3 \%$ of all MRSA isolates were sensitive to imipenem and linezolid respectively.

\section{CONCLUSION}

The present shows that antibiotic resistance is steadily on the rise. It is also quite clear that MRSA is acquiring resistance to drugs like rifampicin, teicoplanin, amikacin, netilmicin and imipenem which were at one time used as an alternative to vancomycin. It is therefore imperative for the medical community to work together to fight against this man-made phenomenon called antibiotic resistance.

\section{KEYWORDS}

MRSA, VISA, VRSA.

HOW TO CITE THIS ARTICLE: Kumar R, Nandan K, Dey S, et al. Methicillin and vancomycin resistance among staphylococcus aureus strains isolated from patients attending tertiary care hospital in Eastern Bihar. J. Evolution Med. Dent. Sci. 2017;6(12): 914917, DOI: 10.14260/Jemds/2017/195

\section{BACKGROUND}

Methicillin Resistant Staphylococcus aureus (MRSA) are implicated in serious infections and nosocomial infection outbreaks. These strains show resistance to a wide range of antibiotics (multi-resistance), thus limiting the treating options to very few agents such as vancomycin and teicoplanin. Therefore, it is clinically crucial to determine

Financial or Other, Competing Interest: None.

Submission 30-12-2016, Peer Review 23-01-2017,

Acceptance 30-01-2017, Published 09-02-2017.

Corresponding Author:

Krishan Nandan,

Department of Microbiology,

Katihar Medical College,

Karim Bagh, Katihar-854105.

E-mail: drknandan@gmail.com

DOI: $10.14260 /$ jemds $/ 2017 / 195$

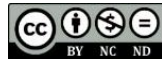

rapidly whether Staphylococcus aureus isolates are methicillin resistant or not and this is of utmost importance for both treatment and control as it requires extensive hygienic precautions to limit the spread of such strains. ${ }^{1}$

Staphylococcus aureus is notorious for its ability to become resistant to antibiotics. Infections that are caused by antibiotic-resistant strains often occur in endemic waves that are initiated by one or a few successful clones. MRSA features prominently in these epidemics. Historically associated with hospital and other health care settings, MRSA has now emerged as a widespread cause of community infections as well. Community-associated MRSA (CA-MRSA) can spread rapidly among healthy individuals. Outbreaks of CA-MRSA infection has been reported worldwide. ${ }^{2}$

The frequency of MRSA infections continues to grow in hospital-associated settings, and more recently in community setting globally. The increase in the incidence of infections due to Staphylococcus aureus is partially a consequence of 
advances in patients care and also of the pathogen's ability to adapt to a changing environment. Infection due to Staphylococcus aureus imposes a high and increasing burden on health care resources. A growing concern is the emergence of MRSA infection in patients with no apparent risk factors. In India MRSA prevalence has increased from $12 \%$ in 1992 to $80.83 \%$ in 1999.2

MRSA emerged as nosocomial pathogen in the early 1960s. Most occurrences were isolated occurring in sporadic outbreaks but in 1970s, an increasing number of large hospital outbreaks were reported in many countries including USA, Europe, Japan, and Australia. Staphylococcus aureus is the predominant organism isolated from surgical wound infections with a prevalence rate ranging from 4.6\%$54.4 \%$ of all Staphylococcus aureus isolations. ${ }^{3}$

Most of these nosocomial infections include bacteraemia, surgical wound infections and pneumonia as well. The problem of MRSA infection is exacerbated by the propensity of the organism to cause cross-infection and its ability to colonize individuals for months or years. Selection pressure for this organism is seen in hospital settings due to intensive use of many antibiotics especially cephalosporins, to which it is resistant. 4

Vancomycin has been regarded as the first-line drug for treatment of MRSA. At the same time, there has been an increase in the use of this antibiotic for other infections as well such as pseudomembranous colitis due to Clostridium difficile and coagulase negative staphylococcal infections in hospitalized patients. When the drug was introduced, it was believed that there would be no resistance to this drug as resistance was difficult to induce. In 1997 however, the first strain of Staphylococcus aureus with reduced susceptibility to vancomycin was reported from Japan. Since then there has been an increase in the number of both vancomycin intermediate Staphylococcus aureus (VISA) and vancomycin resistant Staphylococcus aureus (VRSA). This has led to life threatening infections in both hospitalized and nonhospitalized patients. ${ }^{4}$

Taking into consideration the menace that is MRSA both in hospital and more recently in community settings and also the emergence of VISA and VRSA the study was undertaken to determine the presence of MRSA and VRSA among staphylococcal isolates in Eastern Bihar.

\section{MATERIALS AND METHODS}

This prospective study was conducted from January 2011 to April 2013 with approval from Institutional Ethics Committee. Patients attending various in-patient and outpatient departments of the hospital were inducted into the study. A brief clinical history was obtained from the patient regarding occupation, history of living in institutions e.g. hostels, old age homes etc., personal history regarding standard of hygiene, family history whether other family members were affected and past history.

Urine, pus, blood and vaginal specimens were collected as per standard protocol. Samples were processed and identified as per standard protocol. ${ }^{5}$ Antibiotic sensitivity testing was done by modified Kirby-Bauer's disc diffusion test as per the Clinical and Laboratory Standards Institute (CLSI) guidelines (CLSI, 2008). Commercially available antibiotic discs (hi-Media) were used. Screening for MRSA was done by disc diffusion and agar dilution method.

\section{Screening for MRSA by Disc Diffusion}

The test was performed on Muller-Hinton agar with $4 \% \mathrm{NaCl}$ for oxacillin and without $4 \% \mathrm{NaCl}$ for cefoxitin. Using the same procedure as for AST oxacillin disc $1 \mu \mathrm{g}$ and cefoxitin disc $30 \mu \mathrm{g}$ were used and plates were incubated at $35^{\circ} \mathrm{C}$ for 24 hrs. Results were interpreted as per manufacturer interpretation chart. ATCC 25923 was used as sensitive control. 6

\section{Screening of MRSA by Agar Dilution}

Oxacillin powder was obtained in pure form from Hi-Media laboratories, Mumbai. Commercially available dehydrated Muller-Hinton agar from the same company was used to prepare Muller-Hinton agar to which $\mathrm{NaCl}$ was added to a final concentration of $4 \%$. Amount of antibiotic powder to be added to this medium to make a final concentration of 6 $\mu \mathrm{gm} / \mathrm{ml}$ oxacillin was calculated by the following formula:

$$
\frac{1000}{P} \times V \times C=W
$$

\section{Where}

$\mathrm{P}=$ potency of preparation in relation to base.

$\mathrm{V}=$ volume $(\mathrm{ml})$ required.

$\mathrm{C}=$ final concentration of solution in multiples of 1000 .

$\mathrm{W}=$ weight in mg of antibiotic to be dissolved in $\mathrm{V}$ (volume). ${ }^{7}$

Colonies of the test organism were suspended in $4 \mathrm{ml}$ of sterile normal saline to adjust the turbidity to $0.5 \mathrm{McF}$ arland's standard. The bacterial strains were spot inoculated on the surface of Mueller-Hinton agar containing 4\% $\mathrm{NaCl}$ and 6 $\mu \mathrm{gm} / \mathrm{ml}$ oxacillin using $10 \mu \mathrm{l}$ of bacterial culture suspension. When the inoculum dried up the plates were incubated at $35^{\circ} \mathrm{C}$ for $24 \mathrm{hrs}$.

Interpretation: No Growth - Oxacillin susceptible. Growth - Oxacillin resistant. ${ }^{6}$

\section{Screening for VRSA by Disc Diffusion}

The test was performed on plain Mueller-Hinton agar using $30 \mu \mathrm{g}$ vancomycin disc using the modified Kirby-Bauer's diffusion method. Results were interpreted as per manufacturer interpretation chart. ATCC 25923 was used as sensitive control.6 (CLSI, 2008).

\section{MIC of VRSA by Agar Dilution}

Vancomycin powder was obtained in pure form from HiMedia laboratories, Mumbai. Minimum Inhibitory Concentration (MIC) of vancomycin to Staphylococcus aureus was determined by agar dilution method. The gradient plates of Mueller-Hinton agar were prepared with different concentrations of vancomycin (i.e. 1, 2, 4, 8, 16, 32 and 64 $\mu \mathrm{g} / \mathrm{ml}$ ). The test organisms were suspended in sterile normal saline and the turbidity was matched to 0.5 McFarland's standard. The bacterial strains were spot inoculated on the surface of Mueller-Hinton agar using $10 \mu \mathrm{l}$ of bacterial culture suspension. When the inoculum dried up the plates were incubated at $35^{\circ} \mathrm{C}$ for $24 \mathrm{hrs}$.

Interpretation: $\mathrm{MIC} \leq 2 \mu \mathrm{g} / \mathrm{ml}$ - Susceptible. MIC 4-8 $\mu \mathrm{g} / \mathrm{ml}$ - Intermediate Susceptible. MIC $\geq 16 \mu \mathrm{g} / \mathrm{ml}^{-}$Resistant. ${ }^{6}$ 


\section{RESULTS}

A total of ten thousand eight hundred and six (10806) samples were collected during the study period of which 4482 samples showed growth of various microorganisms. Staphylococcus species grew in 873 samples out of which 240 were Coagulase Negative Staphylococcus (CONS) and 633 were Staphylococcus aureus. These 633 strains of Staphylococcus aureus were taken up for further study.Out of a total of 633 Staphylococcus aureus isolates, 142 (22.4\%) were found to be methicillin resistant, 63 (9.95\%) were VISA and $24(3.79 \%)$ were VRSA. Majority of MRSA and VRSA strains were isolated from the age group 21-30 years, 47/142 (33.1\%) and 13/24 (54.2\%) respectively. MRSA and VRSA infections were seen predominantly in males 79/142 (55.6\%) and $15 / 24(62.5 \%)$ respectively. The male to female ratio for MRSA infection was 1.25:1 and for VRSA 1.66:1. MRSA was isolated more frequently from patients of the Hindu community, 87/142 (61.3\%) whereas VRSA were isolated more frequently from the Muslim community 14/24 (58.5\%). The Hindu to Muslim ratio for MRSA infection was 1.58:1 and for VRSA was 1:1.66.

Most of the MRSA and VRSA were isolated from pus samples followed by urine Table 1. Overall 33.1\% (47/142) of MRSA and 42.8\% (27/63) of VISA were isolated from Surgery Department whereas $41.7 \%(10 / 24)$ of VRSA was isolated from Orthopaedics Department. Majority of MRSA (88.7\%), VISA (74.6\%) and all VRSA were isolated from inpatients department.
Results of oxacillin agar dilution method were in concordance with the cefoxitin disc diffusion method detecting $22.4 \%$ (142/633) of strains as MRSA. The oxacillin disc diffusion method however, detected sixteen additional strains thereby increasing the number of MRSA detected by this method to $25.0 \%(158 / 633)$. Vancomycin DD test detected only 14 strains of VRSA out of a total of 633 strains. The Vancomycin agar dilution test however showed that 24 strains had MIC $\geq 16 \mu \mathrm{g} / \mathrm{ml}$ indicating resistance (VRSA) and 63 strains had MIC ranging from $4-8 \mu \mathrm{g} / \mathrm{ml}$ indicating intermediate resistance (VISA). Table 2.

As with MRSA, VISA and VRSA strain, it showed $100 \%$ resistance to ampicillin, cefuroxime and cefotaxime. $73 \%$ and $75 \%$ of VISA and VRSA were resistant to gentamicin. All VISA strains were sensitive to linezolid and all VRSA were sensitive to imipenem. $88.7 \%$ and $87.3 \%$ of all MRSA isolates were sensitive to imipenem and linezolid respectively. Table 3.

\begin{tabular}{|c|c|c|c|}
\hline Specimen & MRSA (\%) & VISA (\%) & VRSA (\%) \\
\hline Pus & $79(55.7)$ & $34(54.0)$ & $14(58.3)$ \\
\hline Urine & $47(33.1)$ & $18(28.6)$ & $07(29.2)$ \\
\hline Blood & $08(5.6)$ & $07(11.1)$ & $02(8.3)$ \\
\hline Vaginal Swab & $08(5.6)$ & $04(6.3)$ & $01(4.2)$ \\
\hline Total & 142 (100) & $\mathbf{6 3}(100)$ & 24 (100) \\
\hline \multicolumn{4}{|c|}{ Table 1. Isolation of MRSA, VISA and VRSA } \\
from Different Clinical Samples \\
\hline
\end{tabular}

\begin{tabular}{|c|c|c|c|c|}
\hline Method & Resistant (\%) & Intermediate (\%) & Sensitive (\%) & Total \\
\hline Vancomycin DD & $14(2.2)$ & $0(0.0)$ & $619(97.8)$ & 633 \\
\hline Vancomycin MIC & $24(3.7)$ & $63(10.0)$ & $546(86.3)$ & 633 \\
\hline
\end{tabular}

\begin{tabular}{|c|c|c|c|c|c|c|}
\hline \multirow{2}{*}{$\begin{array}{c}\text { Antibiotics } \\
\text { (Potency) }\end{array}$} & \multicolumn{2}{|c|}{$\begin{array}{c}\text { MRSA } \\
(\%) \mathbf{n = 1 4 2}\end{array}$} & \multicolumn{2}{c|}{$\begin{array}{c}\text { VRSA } \\
\text { (\%) n=63 }\end{array}$} & \multicolumn{2}{c|}{ n=24 } \\
\cline { 2 - 7 } & Sensitive & Resistant & Sensitive & Resistant & Sensitive & Resistant \\
\hline Amikacin $(30 \mu \mathrm{gm})$ & $110(77.5)$ & $32(22.5)$ & $57(90.5)$ & $06(9.5)$ & $18(75.0)$ & $06(25.0)$ \\
\hline Ampicillin $(10 \mu \mathrm{gm})$ & $00(0.0)$ & $142(100.0)$ & $00(0.0)$ & $63(100.0)$ & $00(0.0)$ & $24(100.0)$ \\
\hline Cefotaxime $(30 \mu \mathrm{gm})$ & $00(0.0)$ & $142(100.0)$ & $00(0.0)$ & $63(100.0)$ & $00(0.0)$ & $24(100.0)$ \\
\hline Cefuroxime $(30 \mu \mathrm{gm})$ & $00(0.0)$ & $142(100.0)$ & $00(0.0)$ & $63(100.0)$ & $00(0.0)$ & $24(100.0)$ \\
\hline Clindamycin $(2 \mu \mathrm{gm})$ & $87(61.3)$ & $55(38.7)$ & $46(73.0)$ & $17(27.0)$ & $18(75.0)$ & $06(25.0)$ \\
\hline Gentamicin $(10 \mu \mathrm{gm})$ & $63(44.4)$ & $79(55.6)$ & $17(27.0)$ & $46(73.0)$ & $06(25.0)$ & $18(75.0)$ \\
\hline Imipenem $(10 \mu \mathrm{gm})$ & $126(88.7)$ & $16(11.3)$ & $57(90.5)$ & $06(9.5)$ & $24(100.0)$ & $00(0.0)$ \\
\hline Linezolid $(30 \mu \mathrm{gm})$ & $124(87.3)$ & $18(12.7)$ & $63(100.0)$ & $00(0.0)$ & $12(50.0)$ & $12(50.0)$ \\
\hline Teicoplanin $(30 \mu \mathrm{gm})$ & $71(50.0)$ & $71(50.0)$ & $11(17.5)$ & $52(82.5)$ & $00(0.0)$ & $24(100.0)$ \\
\hline Tobramycin $(10 \mu \mathrm{gm})$ & $89(62.7)$ & $53(37.3)$ & $46(73.0)$ & $17(27.0)$ & $12(50.0)$ & $12(50.0)$ \\
\hline & \multicolumn{7}{|c|}{ Table 3. Antibiotic Susceptibility Pattern for MRSA, VISA and VRSA } \\
\hline
\end{tabular}

\section{DISCUSSION}

The present study gives us an indication regarding the occurrence of MRSA, VISA and VRSA in this region. As far as MRSA is concerned different studies have reported different findings. Authors have reported $79.6 \%$ of MRSA from Hyderabad, ${ }^{8} 34.2 \%$ from South India. ${ }^{9}$ The MRSA isolation in our region was found to be relatively low $(22.50 \%$ of all Staphylococcus aureus isolates) as compared to the isolation rates in some other part of India, which is probably because the Medical College is situated in rural area where the organisms are not exposed to as much antibiotic pressure as in the urban areas. For VISA and VRSA Thati et al ${ }^{8} 2011$ reported $4.47 \%$ isolation of VISA and 1.96 which is lower than ours.

Majority of the MRSA, VISA and VRSA strains were isolated from Surgery department followed by Obstetrics \& Gynaecology and orthopaedics. Tyagi A et $\mathrm{al}^{3}$ in 2008 however, reported maximum isolations from Neurosurgery ward followed by Orthopaedics, Paediatric Surgery, and Cardiothoracic Surgery etc. ${ }^{3}$ This discrepancy may be due to the fact that their study was carried out in a super-specialty hospital and also in a different geographic location.

Isolation rate of MRSA, VISA and VRSA in the present study were much higher in indoor patients, as hospital strains are subjected to more antibiotic pressure leading to 
emergence of resistant strains. Majority of MRSA, VISA and VRSA were also isolated from pus samples followed by urine samples and blood and vaginal swabs as reported by other authors also ${ }^{10,11}$ The oxacillin disc diffusion method however, detected sixteen additional strains. This is probably due to the fact that oxacillin DD test may sometime produce smaller zone of inhibition for those strains of Staphylococcus aureus that are hyperproducers of penicillinase enzymes. ${ }^{12}$ These sixteen strains were therefore not considered as MRSA.

Kour $\mathrm{R}$ et al in 2012 have also reported a similar finding in their study on MRSA ${ }^{13}$. They found the oxacillin DD to be inferior to the cefoxitin DD for detection of MRSA. It is quite apparent from all the studies conducted so far and from the present study that antibiotic resistance is steadily on the rise. It is also quite clear that MRSA is acquiring resistance to drugs like rifampicin, teicoplanin, amikacin, netilmicin and imipenem which were at one time used as an alternative to vancomycin, which is more toxic and has to be delivered parenterally, for treating MRSA infection.2,3,10,14 It is very clear from the present study that resistance to antibiotics is high even in Methicillin Sensitive Staphylococcus aureus (MSSA) though lesser to a certain extent than MRSA. Antibiotic resistance remains a problem even in this rural area where majority of the population are employed in agricultural work. It is therefore imperative for the medical community to work together to fight against this man-made phenomenon called antibiotic resistance.

\section{REFERENCES}

[1] Araj GF, Talhauk RS, Simman CJ, et al. Discrepancies between mecA PCR and conventional tests used for methicillin resistant staphylococcus aureus. Int J Antimicrobial agents 1999;11(1):47-52.

[2] Tsering DC, Pal R, Kar S. Methicillin resistant staphylococcus aureus: prevalance and current susceptibility pattern in sikkim. Journal of Global Infectious Disease 2011;3(1):9-13.

[3] Tyagi A, Kapil A, Singh P. Incidence of methicillin resistant staphylococcus aureus (MRSA) in pus samples at a tertiary care hospital, AIIMS, New Delhi. Journal Indian Academy of Clinical Medicine 2008;9(1):33-5.

[4] Sood PL, Taneja J, Mishra B. Methicillin and vancomycin resistant staphylococcus aureus in hospitalized patients. Journal of Global Infectious Disease 2010;2(3):275-83.
[5] Collee JG, Marr W. Culture of bacteria. In: Collee JG, Fraser AG, Marmion BP, et al. (eds) Mackie and McCartney practical medical microbiology. $14^{\text {th }}$ edn. Churchill Livingstone: New Delhi 2006:113-29.

[6] CLSI. Performance standards for antimicrobial susceptibility testing: eighteenth international supplements, M100-S18 2008;28(1):1-179.

[7] Miles RS, Amyes SGB. Laboratory control of antimicrobial therapy. In: Collee JG, Fraser AG, Marmion BP, et al. (eds) Mackie and McCartney practical medical microbiology. 14th edn. Churchill Livingstone: New Delhi 2006:151-78.

[8] Thati V, Shivannavar CT, Gaddad SM. Vancomycin resistance among methicillin resistant staphylococcus aureus isolates from intensive care units of tertiary care hospitals in Hyderabad. Indian Journal of Medical Research 2011;134(5):704-8.

[9] Dhanalakshmi TA, Umapathy BL, Mohan DR. Prevalence of methicillin, vancomycin and multidrug resistance among staphylococcus aureus. Journal of Clinical and Diagnostic Research 2012;6(6):974-7.

[10] Anupurva S, Sen MR, Nath G, et al. Prevalence of methicillin resistant staphylococcus aureus in tertiary referral hospital in eastern Uttar Pradesh. Indian Journal of Medical Microbiology 2003;21(1):49-51.

[11] Sharma P, Vishwanath G. Study of vancomycin susceptibility in methicillin-resistant staphylococcus aureus isolated from clinical samples. Annals of Tropical Medicine and Public Health 2012;5(3):17880.

[12] Peacock SJ. Staphylococcus. In: Borrello PS, Murray PR, Funke G (eds). Topley \& Wilsons's microbiology \& microbial infections. $10^{\text {th }}$ edn. Hodder Arnold, London 2005:771-832.

[13] Kaur R, Oberoi L, Aggrawal A. Comparative evaluation of latex agglutination method and other phenotypic method for detection of methicillin-resistant staphylococcus aureus. Indian Journal of Medical Microbiology 2012;30(2):252-3.

[14] Rajaduraipandi K, Mani KR, Panneerselvam K, et al. Prevalence and antimicrobial susceptibility pattern of methicillin resistant staphylococcus aureus: a multicentre study. Indian Journal of Medical Microbiology 2006;24(1):34-8. 